Article

\title{
Time-Correlated Vortex Tunneling in Layered Superconductors
}

\author{
John H. Miller Jr. * and Martha Y. S. Villagrán \\ Department of Physics and Texas Center for Superconductivity, University of Houston, 4800 Calhoun Road, \\ Houston, TX 77204-5005, USA; mayosuvi@gmail.com \\ * Correspondence: jhmiller@uh.edu; Tel.: +1-713-743-8257
}

Received: 23 May 2017; Accepted: 15 June 2017; Published: 17 June 2017

\begin{abstract}
The nucleation and dynamics of Josephson and Abrikosov vortices determine the critical currents of layered high- $T_{\mathcal{c}}$ superconducting (HTS) thin films, grain boundaries, and coated conductors, so understanding their mechanisms is of crucial importance. Here, we treat pair creation of Josephson and Abrikosov vortices in layered superconductors as a secondary Josephson effect. Each full vortex is viewed as a composite fluid of micro-vortices, such as pancake vortices, which tunnel coherently via a tunneling matrix element. We introduce a two-terminal magnetic (Weber) blockade effect that blocks tunneling when the applied current is below a threshold value. We simulate vortex tunneling as a dynamic, time-correlated process when the current is above threshold. The model shows nearly precise agreement with voltage-current $(V-I)$ characteristics of HTS cuprate grain boundary junctions, which become more concave rounded as temperature decreases, and also explains the piecewise linear $V$-I behavior observed in iron-pnictide bicrystal junctions and other HTS devices. When applied to either Abrikosov or Josephson pair creation, the model explains a plateau seen in plots of critical current vs. thickness of HTS-coated conductors. The observed correlation between theory and experiment strongly supports the proposed quantum picture of vortex nucleation and dynamics in layered superconductors.
\end{abstract}

Keywords: layered superconductor; thin film; superconducting properties; flux vortex; Josephson vortex; Abrikosov vortex; Josephson junction; quantum tunneling; soliton; grain boundary

\section{Introduction}

Both high- and low- $T_{\mathcal{C}}$ superconducting wires, cables, and Josephson devices are spurring advances in energy, medicine, and other sectors of the economy. The critical current density, or $J_{c}$, of a high- $T_{c}$ superconducting (HTS) thin film or coated conductor is often limited by grain boundaries, where misoriented crystallites join at an interface [1-4]. Each grain boundary acts like a Josephson Junction (JJ) or weak link, usually with a width greater than the Josephson penetration length, $w>>\lambda_{J}[5,6]$. The critical current of a wide JJ is limited by nucleation and motion of Josephson vortices. Abrikosov vortices [7], with a radius comparable to the London penetration length $\lambda_{L}$, and Josephson vortices $[6,8]$ of widths $\sim \lambda_{J}$ and $\sim 2 \lambda_{L}$ along and perpendicular to the grain boundary, respectively, carry quantized magnetic flux, $\Phi_{0}=h / 2 e$. After they nucleate, they limit the $J_{c}[1-4]$ by generating a finite voltage and dissipating energy as they move, driven by the interaction between flux and current [9].

A Josephson vortex is a topological flux soliton [10] dual to the charge soliton of a charge density wave (CDW) [11-14]. Josephson [10] and Abrikosov [15-17] vortices can nucleate or depin, in principle, either by either thermal activation or quantum tunneling [18]. In layered high- $T_{\mathcal{c}}$ superconductors, the latter appears to be enabled by short HTS coherence lengths, pancake vortex structures [19], and light vortex masses [20]. Magnetic relaxation rates of trapped flux in $\mathrm{YBa}_{2} \mathrm{Cu}_{3} \mathrm{O}_{7}(\mathrm{YBCO})$, for 
example, decrease with temperature and are nearly temperature-independent when normalized to magnetization [21], suggesting non-thermally-activated depinning of Abrikosov vortices.

Quantum pair creation of Josephson vortices has been proposed for a wide JJ treated as a sine-Gordon model [22]. Tunneling occurs when the applied current tilts the sinusoidal Josephson coupling energy so each newly created soliton-antisoliton pair bounds a lower energy 'bubble' wide enough to balance the positive energy of the solitons, as depicted in Figure 1a. Such tilted sine-Gordon models predict a Zener-like form for the pair production rate [23], $\Gamma \propto F \exp \left[F_{0} / F\right]$, where the force $F$ is proportional to applied current for a JJ. This is formally identical to the rate of Schwinger electron-positron pair production in 1D [24], or CDW soliton pair production [25], but in these latter cases $F$ is proportional to electric field $E$ rather than current. Figure $1 \mathrm{~b}$ illustrates an approach discussed in the following sections, in which phase differences $\phi_{k}$ across HTS grain boundaries for individual layers allow for nucleation of composite vortices consisting of many pancake vortices.

In a CDW, the internal electric field $E^{*}$ created by a charged soliton-antisoliton pair modifies the smooth Zener-like current-voltage $(I-V)$ curve to create a Coulomb blockade threshold field, $E_{T}=E^{*} / 2$, for pair production [26]. Above the threshold, soliton tunneling becomes correlated in time and exhibits phenomena such as coherent oscillations, narrow-band noise, and mode locking [11-14]. In a similar vein, the next sections discuss: (1) a two-terminal magnetic, or Weber, blockade mechanism leading to a threshold current for pair creation of flux vortices; and (2) time-correlated vortex tunneling when the applied current is above the threshold.

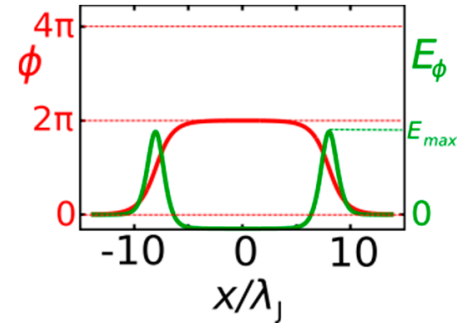

(a)

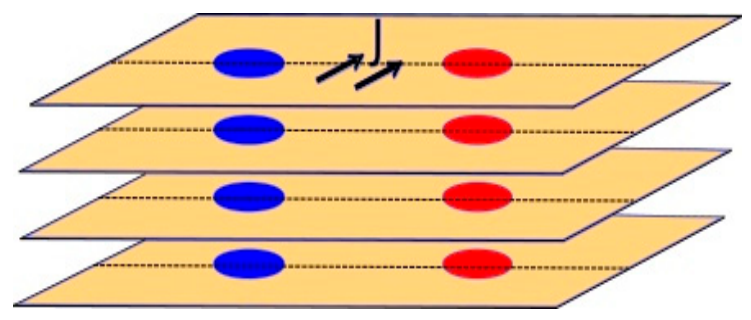

(b)

Figure 1. (a) Nucleation of a soliton-antisoliton pair, showing the phase and energy vs. position (normalized to $\lambda_{J}$ ) along the JJ. The negative energy "bubble" in the middle, ideally, balances the positive energy of a newly nucleated soliton pair; (b) Representation of each Josephson vortex and antivortex as a composite of many pancake vortices straddling grain boundaries within individual high- $T_{\mathcal{C}}$ superconducting (HTS) layers.

\section{Results}

Here we discuss the results of our model, which include the effects of energy on the voltage-current $(V-I)$ curve, due to the magnetic field $B^{*}$ inductively coupling vortices and antivortices. This magnetostatic energy leads to a sharp threshold current for vortex pair creation, and causes vortex tunneling to become correlated in time above threshold. Simulation results are compared with experimental measurements of critical currents in HTS-coated conductors and with voltage-current characteristics of cuprate HTS grain boundary junctions and iron-pnictide bicrystal junctions.

\subsection{Weber Blockade Threshold Current for Vortex Pair Creation}

An HTS grain boundary behaves as a JJ with a periodic Josephson coupling energy vs. $\phi$. Since a $2 \pi$ change in $\phi(x)$ generates a circulating current and vector potential encompassing one flux quantum, $\Phi_{0}$, a $2 \pi$ soliton (antisoliton) in $\phi$ is equivalent to a Josephson vortex (antivortex), e.g., see $[6,8]$. A sufficiently high applied current enables nucleation of a bubble bounded by solitons [22], as shown in Figure 1. Once nucleated, the lower-energy bubble expands as the Lorentz-like force from the current drives the vortex and antivortex apart. Though spatially extended, Josephson vortices are 
extremely light. Using the expression provided by Grosfeld and Stern [27], the mass of a Josephson vortex spanning a 1- $\mu$ m-thick film is estimated to be $\sim 10^{-2} m_{e}$. If, in a layered superconductor, we view a full vortex as a composite of pancake vortices [20] (see Figure 1b), then the mass of each pancake vortex within a single layer is orders of magnitude smaller still.

The two-terminal Weber blockade mechanism proposed here leads to a threshold current for vortex pair creation and is dual to the Coulomb blockade effect that blocks tunneling of electrons [28] or charge solitons [11-14] below a threshold field (see [29] for a three-terminal Weber blockade effect.) Figure 2 illustrates vortex pair creation in a zero externally applied field. The vortices generate a magnetic field with an average value, $B^{*} \sim \beta \Phi_{0} / \lambda^{2}$, linking the vortex and anti-vortex. Here $\lambda=\sqrt{\lambda_{J} \lambda_{L}}$ or $\lambda=\lambda_{L}$ for a Josephson or Abrikosov vortex pair, respectively, and $\beta$ is a dimensionless factor to account for effects of non-uniform field [30], partial field penetration, and/or any vortex overlap. When the film thickness $d$ becomes small, $\lambda$ will be replaced by the thickness-dependent Pearl penetration length $\Lambda$, to be discussed later.

An applied current density $\mathbf{J}$ in a film of thickness $d$ creates an additional self-field, $B_{J} \cong \mu_{0} J d / 2$, which runs just outside the film perpendicular to $\mathbf{J}$ in opposite directions above and below the film, as shown in Figure 2, and can partially cancel $B^{*}$. Once nucleated, the vortices are driven apart by the Lorentz-like force between flux and current.

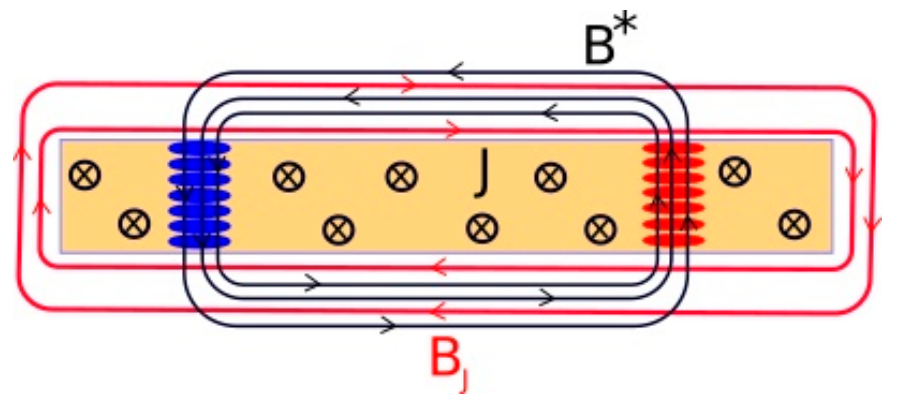

Figure 2. Vortex-antivortex pair in a thin film (end view), illustrating the self-magnetic field $B^{*}$ generated by the vortices and the field $B_{J}$ produced by the applied current $J$. The two contributions, $B_{J}$ and $B^{*}$, partially cancel to reduce magnetostatic energy between the vortices.

Energy conservation prevents vortex pair creation for arbitrarily small applied currents because of the vortices' inductive magnetic energy. Even though $B_{J}$ and $B^{*}$ partially cancel, pair creation is blocked when the integrated magnetic energy between vortices with the pair, $\propto\left(B_{J}-B^{*}\right)^{2} / 2 \mu_{0}$, exceeds that without the pair, $\propto B_{J}^{2} / 2 \mu_{0}$. Equivalently, pair creation cannot occur when the energy difference, $\left(B_{J}-B^{*}\right)^{2} / 2 \mu_{0}-B_{J}^{2} / 2 \mu_{0}=\left(B^{*} / \mu_{0}\right)\left[B^{*} / 2-B_{J}\right]$, is positive, i.e. if $B_{J}<B^{*} / 2$. When the applied current $J$ and self-field $B_{J}$ are sufficiently large that $B_{J}>B^{*} / 2$ however, or when $\theta \equiv 2 \pi B_{J} / B^{*}>\pi$, the former global minimum (vs. $\phi$ ) becomes a metastable state. A bubble bounded by $2 \pi$ solitons (Figure 1) can thus nucleate when $\theta$, proportional to $J$ below threshold, exceeds $\pi$. In the limit, $d>>\lambda$, this yields a threshold pair creation current per unit width given by:

$$
j_{p c}^{0}=B^{*} / \mu_{0}=\beta \Phi_{0} /\left(\mu_{0} \lambda^{2}\right)
$$

When $d$ becomes comparable to or smaller than $\lambda$, Equation (1) will be replaced by an expression that accounts for the thickness dependence of the penetration length, to be discussed shortly. If the nucleated vortex and antivortex are initially separated by a distance $\Delta x$, then the pair creation critical current between vortices can be written as:

$$
j_{p c}^{0} \Delta x \sim \Phi_{0} / 2 L,
$$


where $L \sim \mu_{0} \lambda^{2} / 2 \beta \Delta x$ is the inductance of the flux toroid coupling the vortices. This is essentially the dual of the Coulomb blockade voltage $V_{c}=e / 2 C$ for a small capacitance tunnel junction. Nucleation of a vortex near one edge and antivortex near the other can be treated similarly using the image vortex concept [31].

The critical current vs. thickness, $d$, of HTS-coated conductors usually shows sub-linear behavior and often reaches a plateau, causing $J_{c}$ to decrease with thickness [32-34]. This is readily explained by the Weber blockade mechanism. For a strip of width $w$, the total pair creation current becomes: $I_{p c}^{0}=j_{p c}^{0} w=\beta \Phi_{0} w /\left(\mu_{0} \lambda^{2}\right)$, which is independent of $d$ when $d>>\lambda$. Eventually, however, it is likely that vortex pair creation will be superseded by anisotropic ring nucleation when the film or bulk material becomes extremely thick. As $d$ becomes small the vortices acquire a quasi-2D character and their diameters increase, as predicted by Pearl [35] and directly imaged by Tafuri et al. [36]. When $d \ll \lambda$, the size of each vortex is governed by an effective length, $\Lambda$, known as the Pearl penetration length [35,36], $\Lambda=2 \lambda^{2} / d$. Since the relevant length scale becomes $\Lambda=\lambda$ when $d \gg \lambda$, we use the approximation: $\Lambda \cong \lambda+2 \lambda^{2} / d$. Using $\Lambda$ to replace $\lambda$ in Equation (1) then yields the following expression, as a function of $d$, for the pair creation critical current, $I_{p c}=j_{p c} w$ :

$$
I_{p c}(d)=\frac{I_{p c}^{0}}{[1+2 \lambda / d]^{2}} .
$$

Figure 3 shows a favorable comparison of Equation (3) with measured critical currents vs. thickness of HTS-coated conductors, consistent with the observed plateau effect.

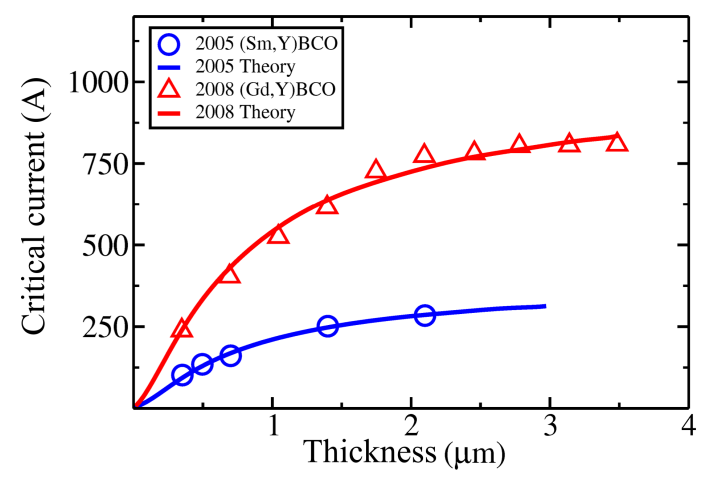

Figure 3. Theory vs. experiment for critical currents of HTS-coated conductors vs. thickness. We use $\lambda=380 \mathrm{~nm} ; I_{p c}^{0}=1030 \mathrm{~A}$ and $I_{p c}^{0}=398$ A to fit the 2008 and 2005 data [34], respectively (see text).

The introduction of insulating $\mathrm{CeO}_{2}$ spacer layers into HTS-coated conductors has been reported to improve the critical current $[32,37]$ vs. thickness behavior by reducing the plateau effect. Our model provides a straightforward interpretation consistent with [37], sufficiently thick spacer layers decouple vortex pair nucleation events on adjacent HTS layers, enabling total critical current to scale with the number of layers. This multi-layer approach is potentially advantageous for HTS magnets constructed with tapes in a pancake geometry, since field lines along the $a b$-plane would be constrained by spacer layers. The next section discusses the dynamics of vortex tunneling above threshold, following a previous model of time-correlated soliton tunneling [11-14] in charge and spin density waves.

\subsection{Time-Correlated, Coherent Tunneling of Josephson Vortices}

In time-correlated single-electron tunneling [28], an applied voltage $V$ across a capacitive tunnel junction increases the displacement charge, $Q=C V$, until it reaches the critical value, $Q=e / 2$, needed to overcome the Coulomb barrier, $(Q \pm e)^{2} / 2 C-Q^{2} / 2 C$. This enables an electron to tunnel through the junction, causing a drop in voltage followed by another increase, and the process repeats many times to cause jerky flow of electric current, $I=d Q / d t$. After $n$ tunneling events, the total 
displacement charge, driven across and through the junction, becomes: $Q=C V+n e$. Time-correlated soliton tunneling in CDWs [12-14] is similar, except that the soliton charge $Q_{0}$ replaces the electron charge $e$. The model combines a sine-Gordon potential, representing CDW pinning, with the solitons' electrostatic energy [26,38]. Following a classic paper on the massive Schwinger model [38], the soliton tunneling model also relates the 'vacuum angle' $\theta(t)$ to the total displacement charge $Q(t)$ and soliton charge $Q_{0}$ using: $\theta=2 \pi\left(Q / Q_{0}\right)$.

In the model proposed here, $\theta$ scales with the applied current $J$ and the resulting self-field $B_{J}$, when $J$ is below threshold, and is proportional to displaced flux when $J$ is above threshold. We define the total displacement flux $\Phi$, after $n$ vortex tunneling/nucleation events, using $\Phi=\lambda^{2} B_{J} / \beta+n \Phi_{0}$. The relation between $\theta$ and $\Phi$ then becomes, $\theta=2 \pi\left(\Phi / \Phi_{0}\right)$, which highlights the duality between flux and charge. We take $\phi_{k}$ to represent phase differences across a junction for individual layers (e.g., Figure 1b), as well as incorporating spatial degrees of freedom. The potential energy for each microscopic degree of freedom $k$ can then be written as:

$$
u\left[\phi_{k}\right]=u_{J}\left[1-\cos \phi_{k}(x)\right]+u_{M}\left(\theta-\phi_{k}(x)\right)^{2},
$$

where the first term is the Josephson coupling energy and the second, quadratic term is the magnetostatic contribution. Figure $4 \mathrm{a}, \mathrm{b}$ shows how the global minimum for the phases switches from the potential well near $\phi \sim 0$ to the one at $\phi \sim \pi$ when $\theta$ crosses above the critical value of $\pi$.
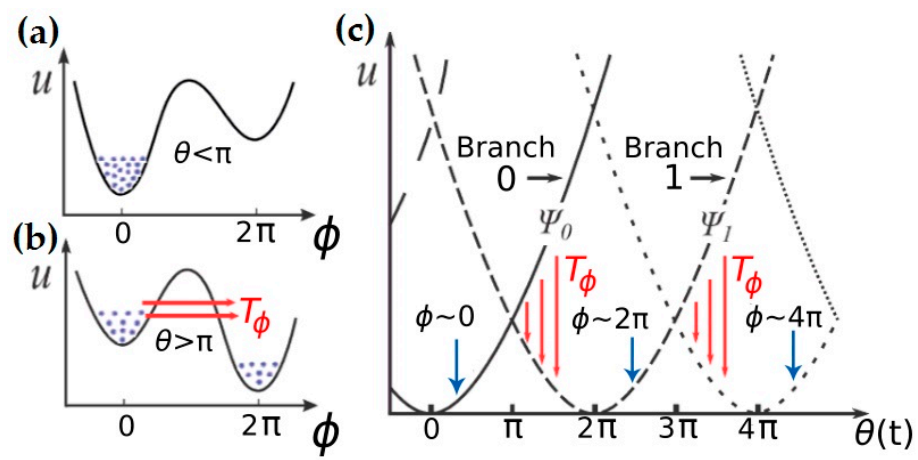

Figure 4. Potential energy plots vs. $\phi$ and $\theta$. (a) The phases $\phi_{k}(x)$ are trapped in the lowest potential well when $\theta<\pi ;(\mathbf{b})$ The original well is no longer a global minimum so the phases begin to tunnel coherently into the new minimum when $\theta>\pi$; (c) Piecewise parabolic potential energy vs. $\theta$ for the various branches representing $\phi \sim 2 \pi n$.

In Figure 4c, Equation (4) is minimized by setting $\phi \sim 2 \pi n$ (dropping the subscript $k$ ) so that $\phi$ is sitting in a potential well (assuming $u_{M} \ll u_{J}$ ). This leads to a series of piecewise parabolic magnetostatic energy plots, $u(\theta) \propto(\theta-2 \pi n)^{2} \propto\left(\Phi-n \Phi_{0}\right)^{2} / 2 L$, as shown in Figure $4 \mathrm{c}$. These are dual to the piecewise charging Coulomb energy curves in time-correlated soliton tunneling in CDWs [11-14], and should not be confused with the Josephson coupling energy. The 'vacuum angle' $\theta$ continually increases with time as the system evolves. Each time a parabola in Figure $4 \mathrm{c}$ crosses the next, at an instability point $\theta=2 \pi\left(n+\frac{1}{2}\right)$, the phases $\phi_{k}$ tunnel coherently into the next well, causing the overall phase to advance by $2 \pi$.

This process repeats itself many times, leading to Josephson-like oscillations and Shapiro steps due to mode-locking with a microwave source. In this picture, although the overall behavior is somewhat jerky, the microscopic degrees of freedom nonetheless flow through the barrier in a continuous fashion, as discussed in [12-14]. The advance with time, of $\theta(t)$ and the phase expectation value $\langle\phi(t)\rangle$, are closely related. Although they only approximately track within a cycle, their correlation becomes precise when time-averaged. Regardless of the detailed shape of the periodic Josephson coupling 
energy, which may or may not be sinusoidal and may include disorder, the emergent behavior as $\theta(t)$ that evolves is often non-sinusoidal.

We now follow [12-14] to model dynamics. We hypothesize that the amplitudes $\psi_{n}$ and $\psi_{n+1}$ for the system to be on branches $n$ and $n+1$ in Figure 4c, respectively, are coupled via coherent, Josephson-like tunneling of microscopic quantum solitons $[39,40]$. We use a matrix element $T_{\varphi}$ to represent this coupling, motivated by Feynman's intuitive derivation [41] of the dc and ac Josephson effects. Advancing $\phi_{k}(x)$ by $2 \pi$ within a finite 'bubble' is equivalent to creating a pair of microscopic solitons. These can either be pancake Josephson vortices [20] with very small masses [27] or even deformations of the Cooper pair wavefunctions within the condensate (see Discussion). We treat the phases $\phi_{k}$ as comprising a quantum fluid, within which they are capable of coherent, Josephson-like tunneling. This picture can presumably be extended to coherent tunneling of Abrikosov pancake vortices [42], which also have small masses [19], or to deformations of their resulting pair wavefunctions.

We use a slightly modified resistively shunted junction (RSJ) model [6], shown in Figure 5, to simulate voltage-current characteristics of an HTS grain boundary junction. This is discussed in Materials and Methods, which also defines the parameters used. Figure 6 shows resulting theoretical voltage vs. current plots for various parameter values. Some of the $V-I$ plots exhibit piecewise linear behavior, which fits neither the classical RSJ model nor a straightforward thermally activated flux flow model. Piecewise quasi-linear behavior is often seen in HTS grain boundary junctions and has largely eluded explanation. Here this behavior is readily explained via the proposed model and two-terminal Weber blockade mechanism. In Figure $6 \mathrm{~b}$ the main effect of increasing $q_{0}$, which corresponds to increasing barrier height and decreasing $\lambda_{\mathrm{J}}$ as temperature goes down, is to increase the degree of rounding in the $V$-I curve.

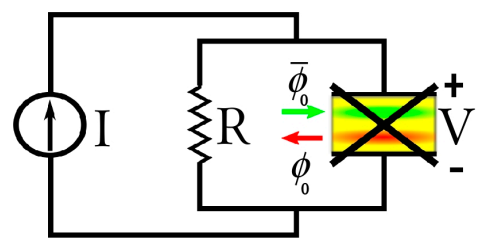

Figure 5. Modified resistively shunted junction model, in which time-correlated tunneling of Josephson vortices is represented by analogy to time-correlated soliton tunneling in charge density wave (CDWs) [11-14].

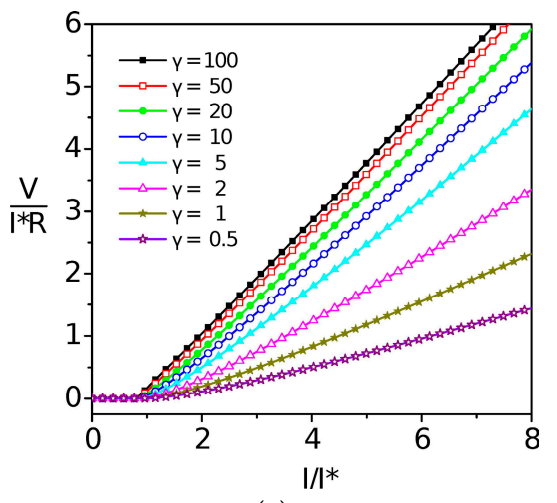

(a)

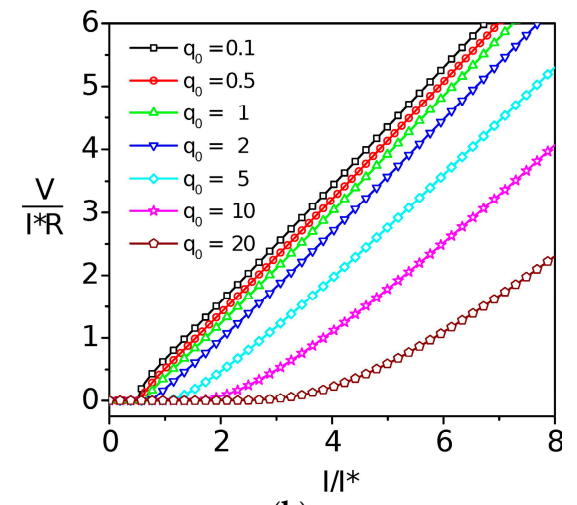

(b)

Figure 6. Simulated voltage-current plots using parameters defined in Materials and Methods. (a) Normalized theoretical voltage-current $(V-I)$ curves for $q_{0}=3$ and several values of $\gamma$; (b) Normalized simulated $V$-I plots for $\gamma=20$ and several values of $q_{0}$, showing the evolution from a rounded Zener-like form to piecewise linear behavior as $q_{0}$ and barrier height decrease. (See Materials and Methods for details.) 
Figure 7 shows comparisons between theory and measured $V-I$ characteristics of an $\mathrm{YBa}_{2} \mathrm{Cu}_{3} \mathrm{O}_{7}$ (YBCO) [43] grain boundary junction at several temperatures. The simulated $86 \mathrm{~K}$ plot in Figure 7 (top) is obtained using the classical resistively shunted junction (RSJ) model in the overdamped limit: $V /\left(I_{c} R_{n}\right)=\sqrt{\left(I / I_{C}\right)^{2}-1}$, without invoking thermal activation. The fact that the $86 \mathrm{~K}$ data fit the classical RSJ model almost perfectly suggests that the effective Josephson penetration length is comparable to or longer than the junction width (short junction limit) due to the small Josephson coupling energy at this temperature. Even at this high temperature there is little or no evidence for concave rounding caused by thermal activation.

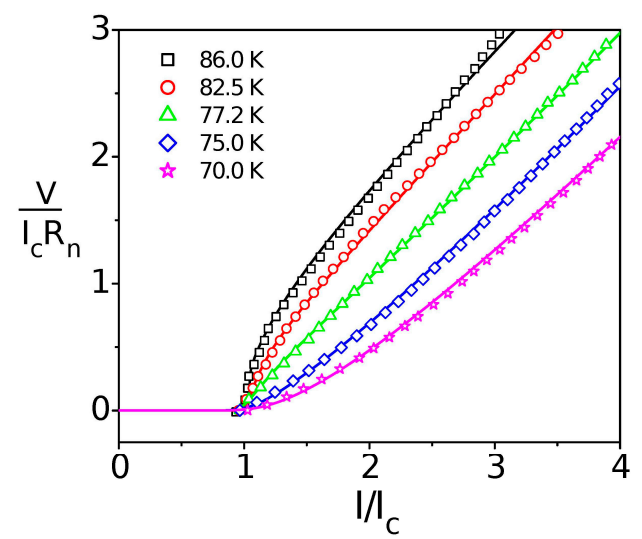

Figure 7. Experiment vs. theory for an $\mathrm{YBa}_{2} \mathrm{Cu}_{3} \mathrm{O}_{7}(\mathrm{YBCO})$ bicrystal junction [43]. Top curves (black): $V$-I curve at $86 \mathrm{~K}$ (dotted line) vs. classical resistively shunted junction (RSJ) model (solid line). Remaining curves: time-correlated vortex tunneling model (solid lines) vs. measured $V$ - $I$ curves (dotted lines) at (top to bottom): $82.5 \mathrm{~K}$ (red), $77.2 \mathrm{~K}$ (green), $75.0 \mathrm{~K}$ (dark blue), and 70.0 K (magenta). (See Materials and Methods, Table 1.)

The theoretical plots for the remaining temperatures in Figure 7, as well as for Figure 8a,b, are obtained via the time-correlated vortex tunneling model (see Materials and Methods), using parameters in Table 1. The theoretical plots (solid lines) show nearly precise quantitative agreement with the experimental data (open symbols). As the temperature is reduced, from $82.5 \mathrm{~K}$ down to $70.0 \mathrm{~K}$, both the experimental and theoretical $V$-I curves show more concave rounding, providing convincing evidence that this is not due to a thermally activated process. The Josephson coupling energy increases with decreasing temperature, causing the characteristic force $F_{0}$ (proportional to $q_{0}$ ) to increase. This increase in $F_{0}$ affects the matrix element $T_{\varphi}$ (Equation (11) of Materials and Methods), and leads to rounded, Zener-like theoretical plots at lower temperatures, in agreement with experiment. Similar quantum fluidic effects may play a role in magnetic relaxation of Abrikosov vortices in bulk YBCO [21], for which $V$-I plots also tend to be quite rounded at low temperatures.

Table 1. Parameters used to generate the simulated $V$ - $I$ curves in Figures 7 and 8.

\begin{tabular}{ccccc}
\hline Figure Plot & $\boldsymbol{I}_{\boldsymbol{c}} / \boldsymbol{I}^{*}$ & $\boldsymbol{w} / \ell$ & $\boldsymbol{\gamma}$ & $\boldsymbol{q}_{\mathbf{0}}$ \\
\hline Figure 7-82.5 K & 0.55 & 1.15 & 50 & 0.5 \\
Figure 7-77.2 K & 0.72 & 1.09 & 45 & 1.7 \\
Figure 7-75.0 K & 1.25 & 1.16 & 40 & 6.5 \\
Figure 7-70.0 K & 1.80 & 1.16 & 30 & 12 \\
Figure 8a & 0.71 & 1.04 & 60 & 1.9 \\
Figure 8b & 1.00 & 1.00 & 12 & 6.0 \\
\hline
\end{tabular}

Figure 8a shows excellent agreement between our quantum model and the $V$ - $I$ curve of an iron pnictide superconducting bicrystal [44], consisting of coupled $\mathrm{SrFe}_{1.74} \mathrm{Co}_{0.26} \mathrm{As}_{2}$ and $\mathrm{Ba}_{0.23} \mathrm{~K}_{0.77} \mathrm{Fe}_{2} \mathrm{As}_{2}$ 
crystals, each $\sim 300 \mu \mathrm{m}$ wide, and thus in the long junction limit. The piecewise linear $V$ - $I$ behavior in Figure 8a also occurs frequently in cuprate grain boundary junctions (e.g., Figure 7, 77.2 K data, and references $[1,45])$. This behavior is analogous to the piecewise linear $I-V$ curve of an ideal Coulomb blockade tunnel junction. Figure 8 b plots our simulation as compared to the measured $V$ - $I$ characteristic of a thallium-based cuprate grain boundary [46], showing good agreement with the data. Table 1 in Materials and Methods shows the parameters used for the simulations and plots.

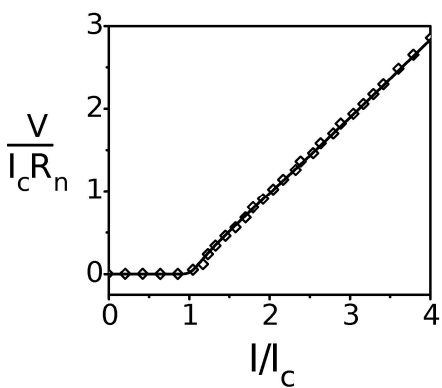

(a)

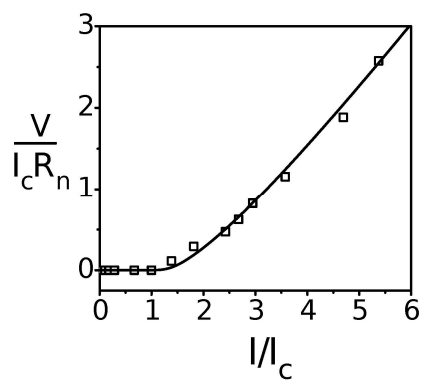

(b)

Figure 8. Further comparisons of model predictions with experimental $V$-I curves. (a) Comparison between time-correlated tunneling model (solid line) vs. $V$-I characteristic (diamonds) of an iron pnictide superconductor bicrystal at $4.2 \mathrm{~K}$ [44]. (b) Model simulations vs. experiment for $V$ - $I$ curve of grain boundary junction in a thallium-based cuprate superconducting film at $77 \mathrm{~K}$ [46]. (See Materials and Methods, Table 1.)

The time-dependent Schrödinger equation (Materials and Methods) and generalized tunneling matrix element $T_{\varphi}$, coupled with the Weber blockade mechanism, provides a simple, yet powerful approach to modeling the dynamics of vortex tunneling. For a uniform junction, the piecewise parabolic curves in Figure 4 and simulations discussed above often predict non-sinusoidal voltage oscillations. The top plot in Figure 9 shows such non-sinusoidal voltage oscillations for a uniform junction. In the remaining plots of Figure 9, we model non-uniformities by representing the junction as 100 domains with a spread in parameters related to coupling energy (see Materials and Methods). The bottom three plots in Figure 9 represent various levels of disorder. The middle two plots show reduced voltage amplitudes and an apparent amplitude modulation effect, consistent with reported subharmonic Shapiro steps [47]. The bottom plot, depicting the most disorder for this series, shows further reduced amplitudes. Voltage oscillations and Shapiro steps are thus expected to become immeasurably small in a sufficiently non-uniform junction.

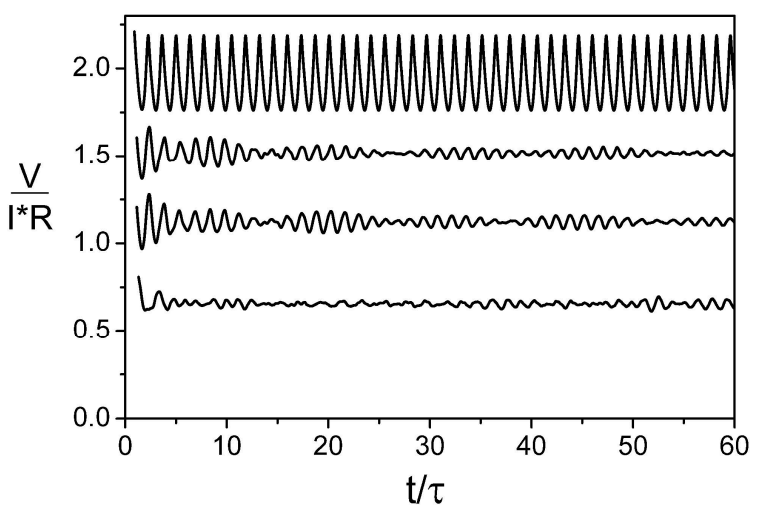

Figure 9. Simulated non-sinusoidal voltage oscillations vs. time (taking $f=I / I^{*}=2.5$ ) for: (top) a uniform junction with $\gamma=40$ and $q_{0}=6.5$; and three nonuniform junctions represented by 100 junctions in parallel. These use the same average $\gamma$ and $q_{0}$ (see Materials and Methods), but with (second from top-to-bottom): $\delta=0.1, k=0.05 ; \delta=0.1, k=0.1$; and $\delta=0.5, k=0.1$. 


\section{Discussion and Conclusions}

This paper considers tunneling of Josephson vortices and, to a lesser extent, Abrikosov vortices. The model, which includes a magnetic Weber blockade effect, shows good quantitative agreement with measured critical current vs. film thickness of HTS-coated conductors, providing a fundamental explanation for the observed critical current plateau. A multilayer approach [32,37] might potentially improve engineering critical currents by decoupling vortex pair creation events in different HTS layers. The spacer layers would also enable flux trapping along the $a b$-plane, which would be especially useful for pancake coil magnets made from HTS tapes. Further work is needed to consider anisotropic vortex ring nucleation in thick films or bulk materials, and to extend the model to include finite external fields. Creation of a dislocation pair [48], for example, may be the energetically least costly process for depinning a vortex lattice in a finite external field. We have also simulated time-correlated tunneling of Josephson vortices. We find nearly precise agreement with measured voltage-current characteristics of HTS grain boundary junctions, which are important for thin film device applications.

A key premise of this paper is that the concept of coherent Josephson-like tunneling should be generalized to include other phenomena with quantum fluidic properties. We view the system as a quantum fluid in which Josephson-like tunneling couples different topological states [49]. While we previously considered tunneling of pancake vortices (Figure 2), an even more microscopic picture is obtained by examining the Bardeen-Cooper-Schrieffer (BCS) ground state [50]: $\psi_{0}=$ $\widehat{\mathrm{A}} \varphi_{0}\left(\mathbf{r}_{1}-\mathbf{r}_{2}\right) \chi_{12} \ldots \varphi_{0}\left(\mathbf{r}_{N-1}-\mathbf{r}_{N}\right) \chi_{N-1, N}$. In this real-space version, $\varphi_{0}$ is the wavefunction for each pair of electrons or holes, $\chi$ is the spin wavefunction, and $\widehat{A}$ is the antisymmetrization operator. The ground state $\psi_{0}$ evolves into a new state, $\psi_{v p}$, containing a vortex-antivortex pair. Each individual pair state evolves into its corresponding vortex-pair-deformed state, $\varphi_{v p}$. Further work is needed to develop a complete microscopic picture. One could, for example, use a representation of operators [51] that operate on the original ground state to create a coherent state description of the vortex pair state.

Beyond improving our understanding of superconductivity and other correlated electron systems, the concepts proposed here could have broad scientific impact in other areas. These include tunneling in quantum cosmology [52-54], perhaps treating the universe as a quantum fluid [55], the theta = pi instability in spontaneous charge-parity $(C P)$ violation [56], and tunneling of atomic bright solitons [57]. Finally, understanding of the quantum behavior of flux solitons could potentially lead to topologically robust forms of quantum information processing or superconducting digital electronics.

\section{Materials and Methods}

Here we discuss simulation of time-correlated vortex tunneling. The voltage across the junction in Figure 5 is:

$$
V=d \Phi / d t=\left(\Phi_{0} / 2 \pi\right) d \theta / d t,
$$

yielding a normal current through the shunt resistance:

$$
I_{n}=V / R=\left(\Phi_{0} / 2 \pi R\right) d \theta / d t
$$

For a grain boundary junction with total current $I$, we define: $\omega=\left(2 \pi R / \Phi_{0}\right) I$. For each 'bubble' nucleation event in which the average phase advances toward the next potential well, the applied current splits into a supercurrent $I_{S}$ and a normal shunt current given by Equation (6). Many such events advance the average phase by $\langle\phi\rangle$ to yield a net supercurrent:

$$
I_{S}=\left(I^{*} / 2 \pi\right)[\theta-\langle\phi\rangle] .
$$

Here $I^{*}=2 j_{p c} \ell$ within a grain boundary region of width $\ell$, comparable to but slightly smaller than $w$ due to the finite width of the vortices. Each time $\langle\phi\rangle$ advances by $2 \pi$, the supercurrent returns close to its previous value, thus preventing it from increasing ad infinitum as $\theta$ evolves with time. 
Using $I_{n}=I-I_{S}$ and introducing the time constant $\tau=L / R$, yields the following equation (also see [12]) for the time rate of change of $\theta$, which is proportional to voltage:

$$
\frac{d \theta}{d t}=\omega-\frac{1}{\tau}[\theta-\langle\phi\rangle]
$$

If the system starts out on branch 0 in Figure $4 \mathrm{c}$, then $\langle\phi\rangle$ will initially be zero. The expectation value $\langle\phi\rangle$ will begin to increase once microscopic degrees of freedom $\phi_{k}$ start tunneling coherently into the next well (Figure $4 \mathrm{~b}$ ) representing branch 1 . Our model represents the amplitudes $\psi_{0,1}$ for the system to be on branches 0 and 1 (or $n$ and $n+1$ ) in terms of coherent amplitudes:

$$
\psi_{0,1}=\sqrt{\rho_{0,1}} \exp \left[i \delta_{0,1}\right]
$$

Here $\rho_{0,1}$ represents the fraction of phases $\phi_{k}$ in the left or right wells, respectively (Figure $4 \mathrm{~b}$ ), corresponding to branches 0 and 1 in Figure $4 \mathrm{c}$. In this picture, $\psi_{0,1}$ are not the superconducting order parameters on opposite sides of the junction but, rather, represent the amplitudes for the phases $\phi_{k}$ to be in either of the two wells in Figure $4 \mathrm{~b}$. The phases $\delta_{0,1}$ are related to Berry phases for vortices, which would, for example, lead to an Aharanov-Casher effect [58].

The expectation value $\langle\phi\rangle$ in Equation (8) is computed by solving the time-dependent Schrödinger equation:

$$
i \hbar \frac{\partial \psi_{0,1}}{\partial t}=U_{0} \psi_{0,1}+T_{\varphi} \psi_{1,0}
$$

Advancing $\phi_{k}(x)$ by $2 \pi$ within a given region, taking $\phi_{k}$ from one branch to the next, is equivalent to creating a pair of $2 \pi$-solitons. The driving force is the energy difference per unit length between potential minima at $\phi \sim 2 \pi n$ and $\phi \sim 2 \pi(n+1)$. When $u_{M} / u_{J} \ll 1$, this force is: $F=4 \pi u_{M} \theta_{n}^{\prime}$, where $\theta_{n}{ }^{\prime} \equiv \theta-2 \pi(n+1 / 2)$. Using the analogy to pair production [24] and following Bardeen [59,60], the effective matrix element for Zener tunneling through the soliton energy gap is estimated to be:

$$
T_{\varphi}(F)=-4 F \lambda \exp \left[-F_{0} / F\right]
$$

Here $\lambda^{-1} \sim \Delta_{\varphi} / \hbar c_{0}+\lambda_{m}^{-1}, \lambda_{m}$ is a mean free path length, $\Delta_{\phi}$ is the soliton energy for a microscopic (e.g., pancake) Josephson vortex, $c_{0}$ is the phase (Swihart) velocity [61], and $F_{0} \sim \Delta_{\varphi}^{2} / \hbar c_{0}$. This expression for $T_{\varphi}$ is similar to the rate of soliton pair production, Zener tunneling of electrons across a bandgap, or Schwinger electron-positron pair production in 1D [24]. Since any negative energy difference within the lower energy 'bubble' is balanced by the positive flux soliton pair energy, the matrix element couples states of equal energy, $U_{0}=U_{1}=U$. Thus, defining $\psi_{0,1}=$ $\chi_{0,1}(t) \exp [-i U t / \hbar]$, the Schrödinger equation (Equation (10)) reduces to:

$$
i \hbar \partial \chi_{0,1} / \partial t=T_{\varphi} \chi_{1,0} .
$$

Below the pair creation threshold, we can write: $\theta=2 \pi I / I^{*}$. In the computer simulations, the variables are put into dimensionless form: $t^{\prime} \equiv t / \tau, f \equiv \omega \tau / 2 \pi$ (proportional to total current $I$ ), $q \equiv \theta / 2 \pi, q_{0} \equiv \theta_{0} / 2 \pi=F_{0} /\left(4 \pi u_{M}\right)$, and $q_{n}{ }^{\prime} \equiv \theta_{n}{ }^{\prime} / 2 \pi=q-n-\frac{1}{2}$. Additionally, $i_{s} \equiv I_{s} / I^{*}=$ $q-p-n$ is the normalized supercurrent, while the normalized voltage is: $v \equiv \frac{d q}{d t^{\prime}}=f-i_{s}$. Finally, setting $\chi_{0}(t)=c_{0}(t)$ and $\chi_{1}(t)=i c_{1}(t)$ in Equation (12), taking $c_{0}$ and $c_{1}$ to be real, yields the following coupled differential equations:

$$
\frac{d c_{1}}{d t^{\prime}}=\left[\gamma q_{n}{ }^{\prime} \exp \left(-q_{0} / q_{n}{ }^{\prime}\right)\right] c_{0}
$$

and

$$
\frac{d c_{0}}{d t^{\prime}}=-\left[\gamma q_{n}{ }^{\prime} \exp \left(-q_{0} / q_{n}{ }^{\prime}\right)\right] c_{1} .
$$


for $q_{n}{ }^{\prime}>0$, where $\gamma=32 \pi^{2} u_{M} \lambda$. These are integrated numerically, taking the initial values to be $c_{0}=1$ and $c_{1}=0$. The phase expectation value is then given by: $\langle\phi\rangle=2 \pi[n+p]$, where $p=\left|c_{1}\right|^{2}$, and the transition is considered complete when $p$ exceeds a cutoff close to one. This process repeats each time the system makes a transition from one branch to the next in Figure 1c. For each current, or value of $f=I / I^{*}$, a time average over several complete cycles is performed to compute $v=(\ell / w)\left(V / I^{*} R\right)$. A similar approach was used previously to compute current-voltage characteristics due to soliton pair production in density waves [12-14]. As in the density wave case, the transition from one branch to the next takes place over a relatively long time interval, suggesting that the phases $\phi_{k}$ 'flow' through the barrier like a quantum fluid rather than tunneling abruptly as a single massive object.

Figures 6-8 show various simulated $V$-I plots, which are compared with experiment in Figures 7 and 8 . Figure $6 \mathrm{~b}$ represents a fixed value of $\gamma=20$ and several values of $q_{0}$. Here, the main effect of increasing $q_{0}$, which corresponds to increasing the energy required to nucleate each vortex-antivortex pair, is to increase the degree of rounding in the $V-I$ curve. As $q_{0}$ becomes significantly greater than one, this rounding causes the 'measured' critical current $I_{c}$ to exceed the nominal Coulomb blockade pair creation critical current, $I_{p c} \cong I^{*} / 2$. For example, when $q_{0}=20$, from Figure $6 \mathrm{~b}$ one can estimate a 'measured' critical current $I_{\mathrm{c}}$ given by: $I_{c} / I^{*} \sim 3$, about a factor of six greater than $I_{p c} / I^{*} \cong 0.5$, but still much smaller than the classical RSJ critical current [6]. Since $\lambda_{J} \sim 1 / \sqrt{u_{J}}$, as the Josephson coupling energy increases, the vortex sizes, $\sim \lambda_{J}$, decrease until they become comparable to $\lambda_{L}$, the London penetration length. Thus, the rounded behavior seen for large $q_{0}$ is also expected for Abrikosov vortex pair creation (provided one minimizes heating effects above the critical current).

Table 1 above shows the parameters used for the simulations that were compared to the experimental results.

The top plot in Figure 9 shows non-sinusoidal voltage oscillations in a uniform junction for a fixed driving current $f=I / I^{*}=2.5$. In the remaining plots of Figure 9 we model non-uniformities by representing the junction as 100 domains in parallel, for which $\gamma$ and $q_{0}$ are pseudo-randomly varied with a uniform distribution within the ranges $\gamma[1 \pm \delta]$ and $q_{0}[1 \pm \delta]$, respectively. The phases in adjacent domains $i$ are coupled by spring-like terms that add to the net force acting on each domain, $k\left[\langle\phi\rangle_{i+1}-2\langle\phi\rangle_{i}+\langle\phi\rangle_{i-1}\right]$, and we apply periodic boundary conditions. The bottom three plots in Figure 9 represent various levels of disorder.

Acknowledgments: The authors acknowledge support by the State of Texas through the Texas Center for Superconductivity at the University of Houston. We acknowledge help in preparing figures from Asanga Iroshan Wijesinghe, and helpful conversations with Venkat Selvamanickam.

Author Contributions: John H. Miller, Jr. conceived the model and performed the simulations; Martha Y.S. Villagrán prepared the figures; John H. Miller, Jr. and Martha Y.S. Villagrán wrote the paper. All authors have read and approved the final version of the manuscript.

Conflicts of Interest: The authors declare no conflict of interest. The founding sponsors had no role in the design of the study; in the collection, analyses, or interpretation of data; in the writing of the manuscript, and in the decision to publish the results.

\section{References}

1. Dimos, D.; Chaudhari, P.; Mannhart, J. Superconducting transport properties of grain boundaries in $\mathrm{YBa}_{2} \mathrm{Cu}_{3} \mathrm{O}_{7}$ bicrystals. Phys. Rev. B 1990, 41, 4038. [CrossRef]

2. Graser, S.; Hirschfeld, P.J.; Kopp, T.; Gutser, R.; Andersen, B.M.; Mannhart, J. How grain boundaries limit supercurrents in high-temperature superconductors. Nat. Phys. 2010, 6, 609-614. [CrossRef]

3. Held, R.; Schneider, C.W.; Mannhart, J.; Allard, L.F.; More, K.L.; Goyal, A. Low-angle grain boundaries in $\mathrm{YBa}_{2} \mathrm{Cu}_{3} \mathrm{O}_{7-\delta}$ with high critical current densities. Phys. Rev. B 2009, 79, 014515. [CrossRef]

4. Hilgenkamp, H.; Mannhart, J. Grain boundaries in high- $\mathrm{T}_{\mathrm{C}}$ superconductors. Rev. Mod. Phys. 2002, 74, 485. [CrossRef]

5. Miller, J., Jr.; Ying, Q.; Zou, Z.; Fan, N.; Xu, J.; Davis, M.; Wolfe, J. Use of Tricrystal Junctions to Probe the Pairing State Symmetry of $\mathrm{YBa}_{2} \mathrm{Cu}_{3} \mathrm{O}_{7-\delta}$. Phys. Rev. Lett. 1995, 74, 2347-2350. [CrossRef] [PubMed] 
6. Barone, A.; Paterno, G. Physics and Applications of Josephson Effect; John Wiley \& Sons: Hoboken, NJ, USA, 1982; p. 529.

7. Blatter, G.; Feigel'man, M.V.; Geshkenbein, V.B.; Larkin, A.I.; Vinokur, V.M. Vortices in high-temperature superconductors. Rev. Mod. Phys. 1994, 66, 1125. [CrossRef]

8. Roditchev, D.; Brun, C.; Serrier-Garcia, L.; Cuevas, J.C.; Bessa, V.H.L.; Milosevic, M.V.; Debontridder, F.; Stolyarov, V.; Cren, T. Direct observation of Josephson vortex cores. Nat. Phys. 2015, 11, 332-337. [CrossRef]

9. Marken, K. Fundamental Issues in High Temperature Superconductor (HTS) Materials Science and Engineering; Woodhead Publishing Limited: Cambridge, UK, 2012; pp. 1-31.

10. Shnirman, A.; Ben-Jacob, E.; Malomed, B. Tunneling and resonant tunneling of fluxons in a long Josephson junction. Phys. Rev. B 1997, 56, 14677. [CrossRef]

11. Miller, J.H.; Ordóñez, C.; Prodan, E. Time-Correlated Soliton Tunneling in Charge and Spin Density Waves. Phys. Rev. Lett. 2000, 84, 1555. [CrossRef] [PubMed]

12. Miller, J.H.; Wijesinghe, A.I.; Tang, Z.; Guloy, A.M. Correlated Quantum Transport of Density Wave Electrons. Phys. Rev. Lett. 2012, 108, 036404. [CrossRef] [PubMed]

13. Miller, J.H.; Wijesinghe, A.I.; Tang, Z.; Guloy, A.M. Coherent quantum transport of charge density waves. Phys. Rev. B 2013, 87, 115127. [CrossRef]

14. Miller, J.H.; Wijesinghe, A.I. The effects of electric and magnetic fields on charge density wave solitons: Possible collective quantum behavior. Eur. Phys. J. Spec. Top. 2013, 222, 1017-1034. [CrossRef]

15. Blatter, G.; Geshkenbein, V.B.; Vinokur, V.M. Quantum collective creep. Phys. Rev. Lett. 1991, 66, 3297. [CrossRef] [PubMed]

16. Ao, P.; Thouless, D.J. Tunneling of a quantized vortex: Roles of pinning and dissipation. Phys. Rev. Lett. 1994, 72, 132. [CrossRef] [PubMed]

17. Wallraff, A.; Lukashenko, A.; Lisenfeld, J.; Kemp, A.; Fistul, M.V.; Koval, Y.; Ustinov, A.V. Quantum dynamics of a single vortex. Nature 2003, 425, 155-158. [CrossRef] [PubMed]

18. Glazman, L.; Fogel, N.Y. Possibility of quantum tunneling of vortices in thin superconducting films. Sov. J. Low Temp. Phys. 1984, 10, 51-52.

19. Chudnovsky, E.M.; Tejada, J. Macroscopic Quantum Tunneling of the Magnetic Moment; Cambridge University Press: Cambridge, NY, USA, 1998; p. 173.

20. Mints, R.G.; Snapiro, I.B. Dynamics of Josephson pancakes in layered superconductors. Phys. Rev. B 1994, 49, 6188. [CrossRef]

21. Xue, Y.Y.; Huang, Z.J.; Hor, P.H.; Chu, C.W. Evidence for collective pinning in high-temperature superconductors. Phys. Rev. B 1991, 43, 13598. [CrossRef]

22. Widom, A.; Srivastava, Y. Electrical engineering implications of the quantized sine-Gordon model. Phys. Lett. A 1986, 114, 337-338. [CrossRef]

23. Dias, O.J.C.; Lemos, J.P.S. False vacuum decay: Effective one-loop action for pair creation of domain walls. J. Math. Phys. 2001, 42, 3292-3298. [CrossRef]

24. Cohen, T.D.; McGady, D.A. Schwinger mechanism revisited. Phys. Rev. D 2008, 78, 036008. [CrossRef]

25. Miller, J.H., Jr.; Cardenas, G.; Garcia-Perez, A.; More, W.; Beckwith, A.W. Quantum pair creation of soliton domain walls. J. Phys. A Math. Gen. 2003, 36, 9209-9221. [CrossRef]

26. Krive, I.V.; Rozhavsky, A.S. On the nature of threshold electric field in quasi-one-dimensional commensurate charge-density-waves. Solid State Commun. 1985, 55, 691-694. [CrossRef]

27. Grosfeld, E.; Stern, A. Observing Majorana bound states of Josephson vortices in topological superconductors. Proc. Natl. Acad. Sci. USA 2011, 108, 11810-11814. [CrossRef] [PubMed]

28. Averin, D.V.; Likharev, K.K. Coulomb blockade of single-electron tunneling, and coherent oscillations in small tunnel junctions. J. Low Temp. Phys. 1986, 62, 345-373. [CrossRef]

29. Morgan-Wall, T.; Leith, B.; Hartman, N.; Rahman, A.; Marković, N. Measurement of Critical Currents of Superconducting Aluminum Nanowires in External Magnetic Fields: Evidence for a Weber Blockade. Phys. Rev. Lett. 2015, 114, 077002. [CrossRef] [PubMed]

30. Kogan, V.G.; Dobrovitski, V.V. Magnetic field of Josephson vortices outside superconductors. Phys. Rev. B 2008, 77, 132504. [CrossRef]

31. Abrikosov, A.A. Fundamentals of the Theory of Metals; North-Holland, Elsevier Science Publishers: Amsterdam, The Netherlands, 1988 ; p. 630. 
32. Foltyn, S.R.; Civale, L.; MacManus-Driscoll, J.L.; Jia, Q.X.; Maiorov, B.; Wang, H.; Maley, M. Materials science challenges for high-temperature superconducting wire. Nat. Mater. 2007, 6, 631-642. [CrossRef] [PubMed]

33. Foltyn, S.R.; Jia, Q.X.; Arendt, P.N.; Kinder, L.; Fan, Y.; Smith, J.F. Relationship between film thickness and the critical current of $\mathrm{YBa}_{2} \mathrm{Cu}_{3} \mathrm{O}_{7-\delta}$-coated conductors. Appl. Phys. Lett. 1999, 75, 3692-3694. [CrossRef]

34. Selvamanickam, V.; Yimin, C.; Xuming, X.; Xie, Y.Y.; Martchevski, M.; Rar, A.; Yunfei, Q.; Schmidt, R.M.; Knoll, A.; Lenseth, K.P.; et al. High Performance 2G Wires: From R\&D to Pilot-Scale Manufacturing. IEEE Trans. Appl. Supercond. 2009, 19, 3225-3230. [CrossRef]

35. Pearl, J. Current Distribution in Superconducting Films Carrying Quantized Fluxoids. Appl. Phys. Lett. 1964, 5, 65-66. [CrossRef]

36. Tafuri, F.; Kirtley, J.R.; Medaglia, P.G.; Orgiani, P.; Balestrino, G. Magnetic Imaging of Pearl Vortices in Artificially Layered $\left(\mathrm{Ba}_{0.9} \mathrm{Nd}_{0.1} \mathrm{CuO}_{2+x}\right)_{m} /\left(\mathrm{CaCuO}_{2}\right)_{n}$ Systems. Phys. Rev. Lett. 2004, 92, 157006. [CrossRef] [PubMed]

37. Wang, X.; Wu, J.Z. Effect of interlayer magnetic coupling on the $\mathrm{J}_{\mathrm{c}}$ of $\mathrm{YBa}_{2} \mathrm{Cu}_{3} \mathrm{O}_{7}$ insulator $/ \mathrm{YBa}_{2} \mathrm{Cu}_{3} \mathrm{O}_{7}$ trilayers. Appl. Phys. Lett. 2006, 88, 062513. [CrossRef]

38. Coleman, S. More about the massive Schwinger model. Ann. Phys. 1976, 101, 239-267. [CrossRef]

39. Maiti, A.; Miller, J.H. Theory of charge-density-wave tunneling. Phys. Rev. B 1991, 43, 12205. [CrossRef]

40. Hermon, Z.; Shnirman, A.; Ben-Jacob, E. Dephasing Length and Coherence of a Quantum Soliton in an Ideal Long Josephson Junction. Phys. Rev. Lett. 1995, 74, 4915-4918. [CrossRef] [PubMed]

41. Feynman, R.P.; Leighton, R.B.; Sands, M. The Feynman Lectures on Physics; Addison-Wesley: Boston, MA, USA, 1965.

42. Grigorenko, A.; Bending, S.; Tamegai, T.; Ooi, S.; Henini, M. A one-dimensional chain state of vortex matter. Nature 2001, 414, 728-731. [CrossRef] [PubMed]

43. Redwing, R.D.; Hinaus, B.M.; Rzchowski, M.S.; Heinig, N.F.; Davidson, B.A.; Nordman, J.E. Observation of strong to Josephson-coupled crossover in $10^{\circ} \mathrm{YBa}_{2} \mathrm{Cu}_{3} \mathrm{O}_{x}$ bicrystal junctions. Appl. Phys. Lett. 1999, 75, 3171-3173. [CrossRef]

44. Zhang, X.; Saha, S.R.; Butch, N.P.; Kirshenbaum, K.; Paglione, J.; Greene, R.L.; Liu, Y.; Yan, L.; Oh, Y.S.; Kim, K.H.; et al. Josephson effect between electron-doped and hole-doped iron pnictide single crystals. Appl. Phys. Lett. 2009, 95, 062510. [CrossRef]

45. Lombardi, F.; Ivanov, Z.G.; Komissinski, P.; Fischer, G.M.; Larsson, P.; Claeson, T. The influence of the top and the bottom grain boundaries on the current transport in $\mathrm{YBa}_{2} \mathrm{Cu}_{3} \mathrm{O}_{7-\delta}$ step-edge Josephson junction. Appl. Supercond. 1998, 6, 437-443. [CrossRef]

46. Dark, C.; Speller, S.; Wu, H.; Sundaresan, A.; Tanaka, Y.; Burnell, G.; Grovenor, C.R.M. Grain boundary properties of Tl-2212 and Tl-1223 thin films. IEEE Trans. Appl. Supercond. 2005, 15, 2931-2934. [CrossRef]

47. Terpstra, D.; IJsselsteijn, R.P.J.; Rogalla, H. Subharmonic Shapiro steps in high-Tc Josephson junctions. Appl. Phys. Lett. 1995, 66, 2286-2288. [CrossRef]

48. Chui, S.T. Depinning, defect creation and quantum tunnelling. Phys. Lett. A 1993, 180, 149-153. [CrossRef]

49. Leggett, A.J. Macroscopic Quantum Systems and the Quantum Theory of Measurement. Progress Theor. Phys. Suppl. 1980, 69, 80-100. [CrossRef]

50. Schrieffer, J.R. Theory of Superconductivity; Benjamin Cummings: San Francisco, CA, USA, 1964.

51. Cahill, K.E.; Glauber, R.J. Density operators for fermions. Phys. Rev. A 1999, 59, 1538-1555. [CrossRef]

52. Atkatz, D.; Pagels, H. Origin of the Universe as a quantum tunneling event. Phys. Rev. D 1982, 25, $2065-2073$. [CrossRef]

53. Linde, A. Quantum creation of an open inflationary universe. Phys. Rev. D 1998, 58, 083514. [CrossRef]

54. Cordero, R.; Rojas, E. Nucleation of ${ }^{(4)} \mathrm{R}$ brane universes. Class. Quantum Gravity 2004, 21, 4231. [CrossRef]

55. Laughlin, R.B. A Different Universe: Reinventing Physics from the Bottom Down; Basic Books, Perseus Books Group: New York, NY, USA, 2005.

56. Tytgat, M.H.G. QCD at theta $\sim$ pi reexamined: Domain walls and spontaneous CP violation. Phys. Rev. D 2000, 61, 114009. [CrossRef]

57. Martin, A.D.; Ruostekoski, J. Quantum dynamics of atomic bright solitons under splitting and recollision, and implications for interferometry. New J. Phys. 2012, 14, 043040. [CrossRef]

58. Elion, W.J.; Wachters, J.J.; Sohn, L.L.; Mooij, J.E. Observation of the Aharonov-Casher effect for vortices in Josephson-junction arrays. Phys. Rev. Lett. 1993, 71, 2311-2314. [CrossRef] [PubMed]

59. Bardeen, J. Tunnelling from a Many-Particle Point of View. Phys. Rev. Lett. 1961, 6, 57-59. [CrossRef] 
60. Duke, C.B. Tunneling in Solids; Academic Press: New York, NY, USA, 1969.

61. Swihart, J. Field Solution for a Thin-Film Superconducting Strip Transmission Line. J. Appl. Phys. 1961, 32, 461-469. [CrossRef] 